\title{
O2O Based, Effective Social Media Marketing Method to Improve Online Exposure
}

\author{
Heejin $\mathrm{Kim}^{1}$, Hyunsook Do ${ }^{2}$ and Byoungju $\mathrm{Choi}^{{ }^{1}}$ \\ ${ }^{1}$ Dept.of Computer Science and Engineering, Ewha Womans University, South \\ Korea \\ ${ }^{2}$ Dept.of Computer Science and Engineering, University of North Texas, United \\ States \\ heejinkim@ewhain.net,hyunsook.do@unt.edu,bjchoi@ewha.ac.kr
}

\begin{abstract}
Recently, social media has been used as an independent marketing tool, and as result, the needs for platforms or tools to manage the data collected by social nelmorks are greatly increasing. To date, many marketing techniques that utilize social media have been proposed, but no research has been done on the problem of helping marketers systematically choose appropriate marketing methods that engage both online social networks and customer experience with offline events. To address this problem, we propose an O2O (Online to Offline) based social-media marketing approach, uLike, which collects customer-experience focused data from offline events to online media in real-time and helps advertise products or brands more easily. To evaluate our approach, we conduct an on and offline marketing-event experiment. Our results show that the approach can be more effective and efficient for increasing the exposure of online advertisements than the traditionalsocial media marketing approaches.
\end{abstract}

Keywords: O2O, Social netwarking servite, Social media marketing, NFC

\section{Introduction}

Along with rapidly growing smart devices, social networking services (SNS) such as Facebook, Twitter, and Instagram have greatly contributed to creating various marketing products and to developing fechnologies that support SNS. These social media options have affected our daily kves in many ways, such as how to interact with other people and how to manage our personal data, and now, it is hard to imagine our everyday lives without social media U]. Because social media can collect and maintain users' personal interests, preferences, and lifestyles, companies can utilize this information for their marketing strategies [2]. Further, social-media platforms allow users to create and distribute bidirectional information that can add value to a company's marketing products, so the technology has begun to emerge as an effective marketing tool [3-4].

Social-media marketing is defined as the methods for advertising products, services, or brands using the internet, by attracting the interest of groups of people who discuss them, make suggestions about them, etc. online. Hersant [5] provides another definition of social-media marketing as targeting specific customers and engaging them with something that direct, memorable and meaningful to them. These definitions indicate that social-media marketing is a means to draw targeted customers' spontaneous participation; to promote the company's brands and products by using social media; and, finally, to get customers' attention. It is reported that many companies make effective use of SNS to keep up with this trend and that $96 \%$ of the individual business that use the social media for their businesses are adopting social-media marketing [6].

${ }^{1}$ Byoungju Choi: Corresponding author 
In order to maximize effects of world-of-mouth through social media, it is essential to obtain the contents that include customer-experience with specific products and brands offline and to post them through online media so as to meet the needs of customers in real-time [7-8]. However, viral marketing is still being implemented mainly based on online channels because of the lack of effective services and methods to support socialmedia marketing in conjunction with offline [9]. To address this issue, in this paper, we propose a social-media marketing approach that can link offline customer- experience and actions online.

The main contributions of this paper are as follows:

- We proposed an O2O-based marketing method that is linked with social media using Near Field Communication (NFC) technology $[10,11]$ that provides a realtime connection between offline events and online media. With this method, users can post personalized contents to their SNS automatically when they use the NFC technology.

- Based on the proposed method, we developed the social media marketing service uLike [12], which allows customers to conveniently share their experiences by adding comments about and photos from real offline events to their SNS services. Further, companies can obtain useful CRM (Customer Rêlationship Management) information and these data can be utilized to improve matketing strategies.

- We evaluated our method with case studies that were applied to real marketing events and then presented the results for the case studies. Throygh these studies, we demonstrated that our method can be more effective and efficient to increase the online exposure effect and can be more useful to increase customers' participation rate when compared with existing social media marketing methods.

The rest of the paper is organized as follows. Section 2 introduces the background. Section 3 describes the system architecture of litike and the proposed approach, our experiences with applying an eafly yersion of usike to real marketing events, and the lessons learned. Section 4 presents our experment, including design, results and analysis. Section 5 discusses our results and their implications. Finally, Section 6 concludes this paper with a summary and discussion of future work.

\section{Background}

\subsection{Social Media Marketing in $\mathrm{O} 2 \mathrm{O}$}

O2O (Online to Off(ine or Offline to Online) has emerged as an important trend of corporate strategies. According to Rampell [13] who introduced the concept of O2O for the first time, the key of idea was to find consumers via online and bring them to offline stores. In this 020 mode, it is possible to maximize synergies by utilizing social media. It is becatsel social media provides real-time services, makes bidirectional communication available, and helps spread information farther and faster [3-4]. Social-media-based marketing is able to get more customers to participate in the offline marketing events and 10 share their thoughts and opinions about products [14]. Moreover, social media can reach potential customers indirectly through the fans and followers (e.g., Twitter: retweet and Facebook: like). Using these advantages, marketing with social media can increase the product or brand image's exposure effect when users' experiences and reviews are uploaded and shared among people who are interested in the product [7].

Many companies have been tried to combine social media with other marketing channels (e.g., traditional media, and web pages). One of the examples is a Diesel Cam promotion [15], which was held at the Diesel store in Madrid, Spain, in March 2010. This offline marketing event was linked with online SNS, and during this event, people could put on the store's clothes and share photos on Facebook. This kind of approach can be effective for drawing the customers' direct participation and responses because people can 
provide customized contents (e.g., postscripts, and opinions from their experiences) based on real-time experiences [9].

The necessities for marketing methods and tools that can share, collect, and analyze social data from the customer's real experiences after people post on the online social media is emerging, but, thus far, no suitable method has been available. Further, no research has considered approaches that combine social-media marketing with $\mathrm{O} 2 \mathrm{O}$. Thus, we believe that this paper adds important advances to the social-media marketing based on $\mathrm{O} 2 \mathrm{O}$.

\subsection{Technologies for $\mathbf{O} 20$}

Advances in technologies such as GPS (Global Positioning System), Beacon, QR (Quick Response) code, and NFC have led to development of new types of services and methods based on $\mathrm{O} 2 \mathrm{O}$ [16]. Among these technologies, recognition technology is that serves to connect customers in real time at an offline store as well as provides information about the product recognized.

NFC, an advanced form of Radio Frequency Identification (RFID) technology, enables communication among electronic devices at close proximity to exchange information in a wireless manner [10]. Although NFC was standardized in 2003, it has gained more popularity since it supported android mobile phones. With a mobile device that has NFC, users can access any information or service they want by utilizing NFC tags that are installed at various everyday places, such as shopping malls/coffee shops, and restaurants. In August 2014, Apple Inc. officially released a mobile payment service called Apple Pay. As such, NFC can be utilized as a communication protocol for mobile payment applications [16]. In this paper, we use NFC technology as an individual identifier for linking online and offline

\section{3. uLike Approach}

In this section, we describe an infrastructure that supports the uLike approach and provide details about the urike techniẹte, including two experiences with applying $u$ Like to real-world events.

\subsection{System Architecture}

The infrastructure that supports the uLike approach consists of four components: a PC with uLike and NFC devices, posting devices, servers, and databases. Typically, an NFC tag is provided to user $m$ the form of a wristband in order to support portability, and an NFC reader is connected to a PC through the USB port. Three different devices that facilitate online posting activities can be used with this infrastructure: a mobile phone with NFC a reader stand, and a kiosk. The posting server collects all the materials that participants upload, and then, it transmits the collected data to SNS servers in real time. The web server manages the web pages for a back-office that provides administrative information and supports uLike. To systematically organize and maintain the data collected from event participants, the infrastructure provides five different databases. We describe these databases in detail:

- Member Database: Participants' basic information that is obtained from the SNS (i.e., name, gender, email address, and number of SNS friends)

- Content Database: Content information to be used during the events (i.e., duration of the event and content images)

- Posting-History Database: Posts that are uploaded by the participants (i.e., photos that participants post)

- Survey Database: Data for the results (i.e., favorite color) obtained from the surveys completed by participants 
- Statistics Database: Tracking data for the uploaded posts (i.e., comments, likes, and shares) as well as CRM statistics, such as the number of posts and comments, customer lists, like hits, and other demographic information.

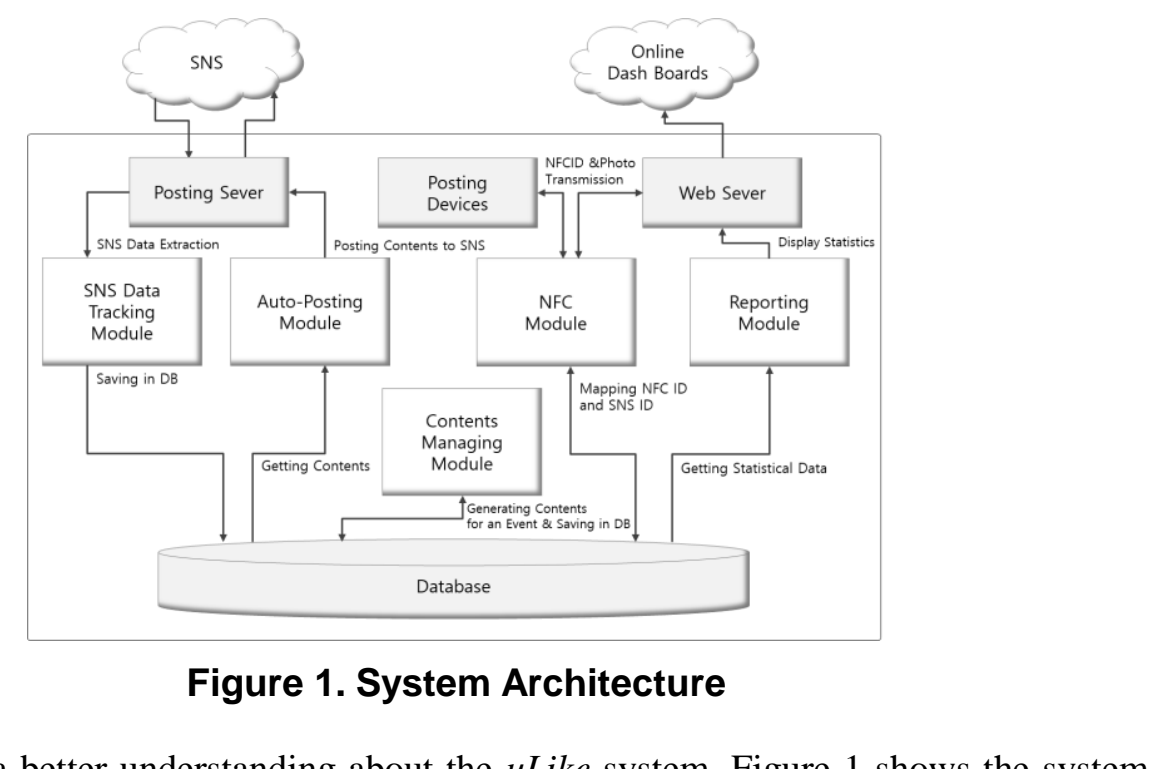

To provide a better understanding about the $u$ Like system, Figure 1 shows the system architecture; there are five main modules shown in the center, white boxes, and relationships among these modules and other components of the $u$ Like infrastructure are illustrated. We describe these modules in detail:

- SNS Data-Tracking Module: This module tracks data concerning uploaded SNS posts.

- Auto-Posting Module: This module automatically posts, on multiple SNS platforms (e.g., Facebook, Twitter, Instagram, ete), the contents that are retrieved from the content DB. This module provides an SNS-posting demon that posts data to the SNS server everyminate.

- NFC Module. This modulevenables the users to map the NFC tag IDs that are read from the posting devices onto their own SNS IDs, and then, the value of the mapping information is saved in the database.

- Reporting Modute. This module displays various real-time statistical data (e.g., posting statistics, survey statistics, and user statistics) on the back-office dashboards than senerated from information stored in the statistics DB and from CRM information.

- Content-Managing Module: This module generates event contents (e.g., an event logo, an event webpage-link, and an event video clip) by using content templates from the content DB.

\subsection{The Use of uLike: A Typical Scenario}

The scenario starts with the participants' visit to the offline marketing-event site. It is assumed that all participants have their own SNS accounts. This assumption is realistic because most people will have their own accounts in the future. Figure 2 shows the flowchart of main activities that are required to perform such a scenario and how these activities are related to each other. Three main activities are involved with uLike-based events: interlinking SNS with the NFC tag, real-time multi-posting, and monitoring and management. The following subsections describe each activity in detail. 

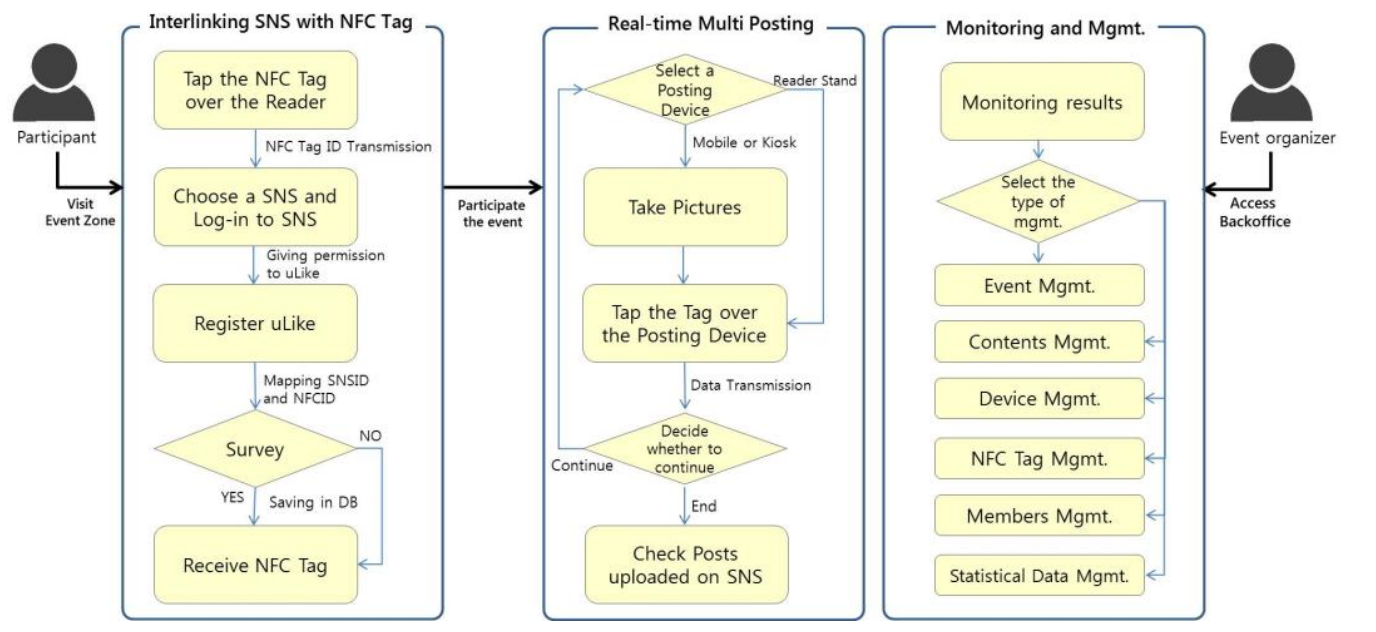

Figure 2. Flowchart of uLike

\subsubsection{Interlinking SNS with the NFC Tag}

Once participants visit the event, they receive an NFC tag that is configured with an SNS account. Figure 3 shows the process of linking the participants' SNS accounts with the NFC tag. First, the participants tap the NFC tag over the NFC reader. Then, the participants' NFC tag IDs are transmitted to the web server Next, the participants can choose the SNS account that they want to use When the participants log into their SNS account, they go through the uLike service's pernission step that authenticates and authorizes an SNS API. This permission step is requined for first-time users of the service. If people have utilized the uLike sepvice before, they can skip this permission step. Next, they should sign up by giving basie information about the SNS account. As an optional process, the participants can take a suryey. The questionnaire results are saved in the database and made availabie for the statistical analysis.

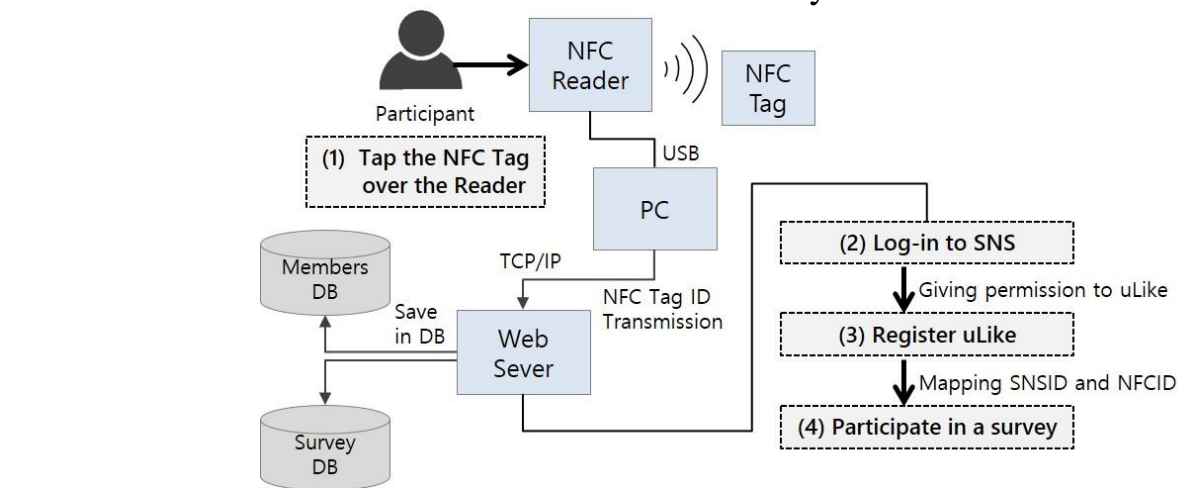

Figure 3. Process of Interlinking with NFC Tag

\subsubsection{Real-Time Multi-Posting}

Once the participants have the NFC tag linked with their SNS accounts, they can take part in real-time, multi-posting events by using the posting devices that are tagged to the provided NFC tags. To do so, the participants select a posting device. If the participants select the reader stand, they can tap the NFC tag over the reader without taking pictures. The preconfigured contents (e.g., logo, sign, and homepage web-link) and the NFC tag ID are immediately transmitted to the server and posted on the SNS. If the participants select either a mobile device with NFC or a kiosk, they can take pictures to post. When using a 
mobile device, participants take pictures with the help of the event staff members who carry a mobile device with the uLike application installed. Afterwards, the participant's NFC tag is tagged on the backside of the mobile phone. With the kiosk, on the other hand, the participants are required to take their own pictures to tag. Although each photo can only be identified once with a tag, multiple tags can be identified simultaneously, and those tags can be posted on multiple SNS platforms at the same time. If individuals want to continue participating in the event, they can tap the tag over any other posting devices. When the participants check the results for the event participation, they can verify the preconfigured contents and photos that were uploaded on their SNS. The posts are shared and spread among friends via the SNS. The process is summarized in Figure 4.

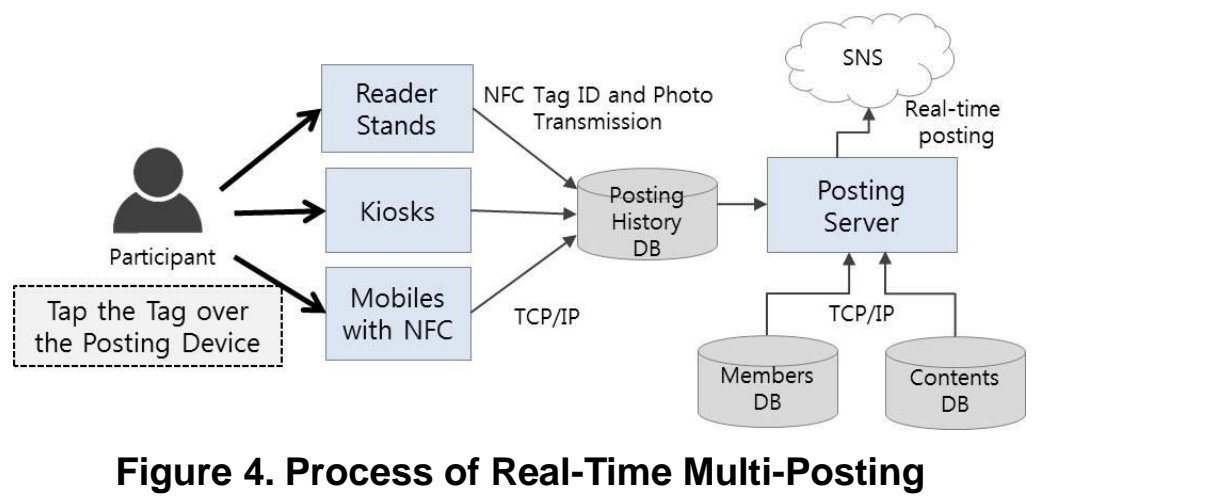

\subsubsection{Monitoring and Management}

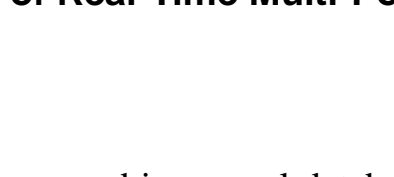

All data collected during the event are saved in several databases. The event organizer can access online back-office and monitor real-time activities in order to manage the events, devices, contents, NFC tags, and statistics. All monitored data are categorized by event, SNS, type of posting device, and date, and the data displayed on the dashboards. The process is summarizedin Figure 5. We describe these activities, in detail, as follows:

- Event management; registration and modification of customers' events as well as management of event information (i.e., event duration and location).

- Content management: registration, modification, and deletion of the posted contents for an eventon posting devices as well as configuring the logo images and statements for the posted contents.

- Device management: registration and management of the posting devices for each event.

- NFC Tas management: management of tag-issuance information and of tag UID inforrhation.

- Member management: management of user information (i.e., gender, age group, and SNS platform).

Statistical data management: management of statistics regarding many different aspects of the posts, shared data concerning the posts, and survey results in realtime (i.e., number of posts by age groups, by posting devices, and by event contents). 


\subsubsection{Experience 2. 2013 Brazil Game Show}

At the Brazil Game Show (BGS) ${ }^{2}$ held in Sao Paulo during October 25-29, 2013, uLike was utilized for the event that operated at three booths to exhibit Korean video games: TalesRunner, Bn1944, and NurigoGames. Brazil Game Show, known by the initials BGS, is a yearly South American video-game convention and is the largest gaming convention in Latin America. Using the received tags, the participants could post photos or content about each game to SNS platforms and could enter the raffle to win some prizes. This event scenario was as follows.

- Step 1: After logging in to the participants' SNS account at each booth, they receive a bracelet type of the NFC tag that is linked with their SNS account.

- Step 2: The participants tap the tag over the displayed reader stands at the booth or take pictures with the help of the event staff members who carry a mobile device and tap the tag over the device.

- Step 3: The preconfigured photos or content is uploaded to the participants' SNS, and the participants can check to see if they win prizes.

The total number of participants was 1,152 , consisting of 312 peopl at the Tales Runner booth, 543 at the Bn1994 booth, and 297 at the NurigoGames booth. Table 2 shows the posting results collected from each booth. Two types of posting devices were used at this event: reader stands and mobile phones. Using these devices, the number of postings as well as the number of the responses and likes was counted in order to determine how many Twitter and Facebook posts were created by the participants along with how much interest uploaded posts received. The number of Twitter posts was 21 ; the number of Facebook posts was 1,810; the number of Facebook likes was 2,465; and the number of Facebook comments was 726.

Table 2. Results for Experiment 2

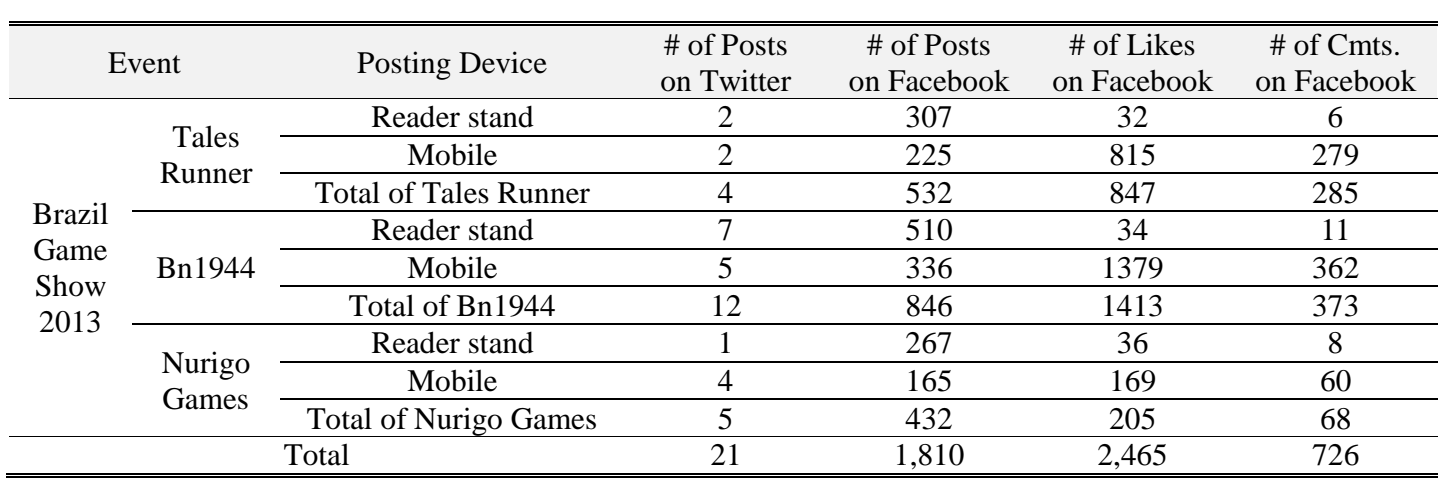

\subsubsection{Lessons Learned from the Two Experiences}

We have learned several important lessons from these experiences. First, we have found hat uLike is helpful in encouraging people to participate in the events. We speculate that $u$ Like's ease of use attracted more people.

Second, we have found that SNS friends showed high interest in the content that was posted using uLike and that they responded more actively. Even though the content that was posted by the participants included advertisements for a company or a brand, friends reacted to the posts with a lot of responses and likes. One possible reason for this is that the contents were voluntarily posted on the participants' SNS platforms.

Third, because uLike allows us to automatically collect statistical data about the SNS posts through uLike, we can measure the event's performance, such as event key performance indicators (KPIs; e.g., PR and advertising effect) by analyzing the collected

\footnotetext{
${ }^{2}$ http://www.brasilgameshow.com.br/
} 
data. We believe that, by understanding the event's performance, organizers can improve their marketing strategies.

\section{Empirical Study}

Using the uLike system and the infrastructure explained in Section 3, we conducted a controlled experiment to evaluate the proposed method. To assess our approach, four research questions were investigated.

- RQ1: Is the uLike method more effective (i.e., the number of posts) for increased online exposure compared to an existing social-media marketing method?

- RQ2: Is the uLike method more efficient (i.e., the number of posts per hour) for increased online exposure compared to an existing social-media marketing method?

- RQ3: Does the uLike method have a higher viral effect (i.e., the number of comments) than an existing social-media marketing method?

- RQ4: Is the uLike method useful for improving the customer's social engagement?

\subsection{Variables and Measures}

To investigate our research questions, we manipulate one indepêndent variable:

- A Social Media Marketing Method: Participants used the uLike method and a nonuLike method while they participated in the marketing event

We also measured the following dependent variables:

- Effectiveness: Two values were measured: the number of posts (how much content is posted on SNS platforms) and the number of fans (how many fans on the official SNS page have been increased

- Efficiency: The efficiency gan also be measured with two values: the number of posts per participant and the mumber of fans on the official SNS page per hour.

- Viral effect: This can be measured by viral factors, such as the number of comments, shares, and likes. By aramining these numbers, we can understand how the marketing follow-up activities vere actively performed.

\subsection{Experimental Procedure}

The experimental design includes several steps. Figure 6 shows the experiment's sequence. The details are provided in the following subsections: 


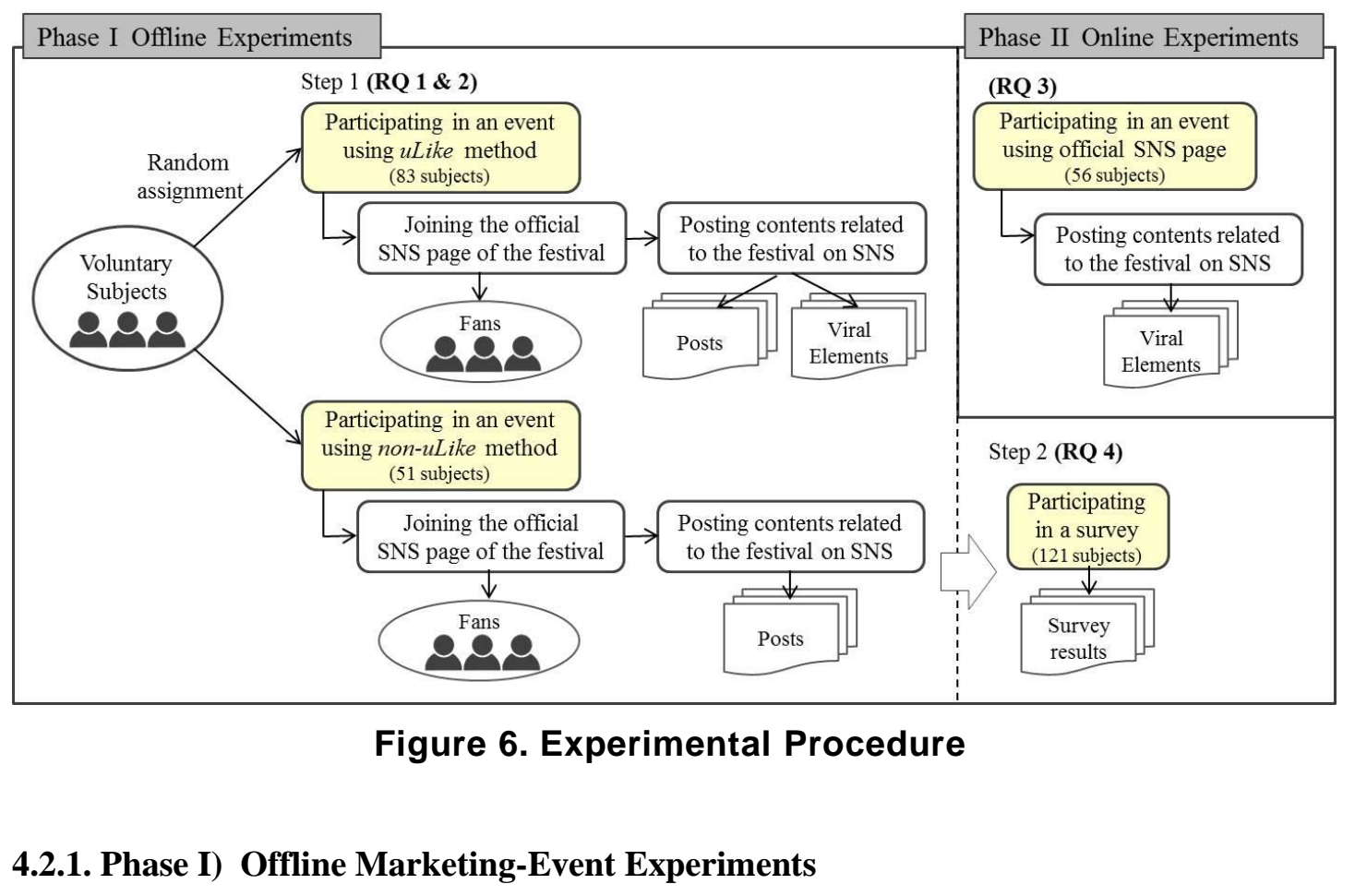

There were two steps in this phase. First, we conducted offline marketing-event experiment using uLike and non-uLike methods. Second, we performed a post-research survey to obtain feedback from the participants.

Step 1. Participating in a markefing event experiment using uLike and non-uLike methods: The pre-promotion event for the Hwacheon Sancheoneo Ice Festival 2014 was selected as the offline marketing event for this experiment. This event's goal was to attract attention for the festival that ran from Jan. $4^{\text {th }}$ to $26^{\text {th }}$ in 2014 , and to promote the event through SNS platforms. The Hwacheon Festival has been held in January every year since 2003 to adverise ice fishing activities in Hwacheon-gun, South Korea. This festival is one of the seven wintermysteries introduced by $\mathrm{CNN}$, and it attracts more than one million people. We decidec to conduct this experiment around the Gangnam Station area in Seour because Gangman Station is one of the most crowed areas in South Korea, thus it was easy to find volunteers who could participate. We set up two booths around Gangnam Station: one for the experiment with uLike and the other one with a non-uLike method. Voluntary participants were randomly assigned to each booth. Table 3 summarizes infornation about Step 1 for Phase I of the experiment.

\section{Table 3. The Experiment Information for Step 1 in Phase I}

\begin{tabular}{ll}
\hline \hline Experiment Event & Pre-promotion event for the 2014 Hwacheon Sancheoneo Ice Festival 2014 \\
\hline Experiment Date & Dec. 14, 2013, from 11 am-3 pm \\
\hline Experiment Place & Two booths around Gangnam Station in Seoul, South Korea \\
\hline Experiment Subject & 134 volunteers around Gangnam Station \\
\hline Experiment Method & The marketing event using uLike and non-uLike methods \\
\hline
\end{tabular}

Participants were asked to join the official SNS page for the festival, but it was not required. Next, the participants were required to post festival-related content on the SNS site. People could post various content types, such as text, video clip, and photo. The participants received a giveaway, such as a beverage coupon, after they finished the required tasks. The $u$ Like participants were required to obtain an NFC tag in advance to perform the experiment. After connecting the tag and their SNS by logging into Facebook, related content (e.g., festival date and venue) was posted by simply tapping the 
connected tag to the reader stand. On the other hand, the non-uLike participants just needed their own mobile phones instead of using NFC tags. In order to post content on a SNS, the SNS applications should be installed on people's mobile devices. The non-uLike participants uploaded information by directly generating content in various ways, such as recording their experiences, taking pictures, and linking to a video clip from the web site after logging into Facebook.

Table 4. Assessment Form

\begin{tabular}{|c|c|}
\hline $\begin{array}{l}\text { Assessment } \\
\text { Items }\end{array}$ & Details \\
\hline Innovativeness & $\begin{array}{l}\text { Do you think that automatic posting using } u \text { Like is innovative? } \\
\text { 1) Not at all innovative 2) Little innovative 3) Average 4) Innovative 5) Very innovative }\end{array}$ \\
\hline Usability & $\begin{array}{l}\text { Do you think that it is convenient to upload posts on your SNS using the } u L i k e \text { ? } \\
\text { 1) Very inconvenient 2) Inconvenient 3) Average 4) Convenient 5) Very convenient }\end{array}$ \\
\hline Attractiveness & $\begin{array}{l}\text { Do you like your experience with the use of } u \text { Like in terms of its real time posting } \\
\text { ability to SNS? } \\
\text { 1) Not at all attractive 2) Little attractive 3) Average 4) Attractive 5) Very attractive }\end{array}$ \\
\hline $\begin{array}{l}\text { Brand-Image } \\
\text { Promotion }\end{array}$ & $\begin{array}{l}\text { Do you think that the company's brand image will change by employing the uLike } \\
\text { system to their marketing event? } \\
\text { 1) Very negative 2) Negative 3) Average 4) Positive 5) Very positive }\end{array}$ \\
\hline $\begin{array}{l}\text { Future } u \text { Like } \\
\text { Use }\end{array}$ & $\begin{array}{l}\text { Are you willing to participate in "Sancheoneo Festival" which uses the uLike } \\
\text { system? } \\
\text { 1) Not at all 2) A little 3) Average 4) Will hing to 5) Very willing to }\end{array}$ \\
\hline $\begin{array}{c}\text { Overall } \\
\text { Satisfaction }\end{array}$ & $\begin{array}{l}\text { How are you satisfied with uLike system overall? } \\
\text { 1) Very dissatisfied 2) Dissatisfied 3) Average 4) Satisfied 5) Very satisfied }\end{array}$ \\
\hline
\end{tabular}

Step 2. Participating in a survey: We performeda post-research survey to obtain feedback from the participants who used the uLike and non-uLike methods to see whether uLike was useful to encourage people to participate in the events. We asked six questions of both groups as shown in Table 4. Because the non-uLike group did not have any prior experience with $u$ Like, before those individuals took the survey, we explained the uLike method using story boards

\subsubsection{Phase II) Online Marketing-Event Experiments}

During this phase, we conducted an online marketing-event experiment. The purpose of this experiment, was to see, whether the uLike method had higher viral effects than the non-uLike method. To measure the viral effects, all the data that were shared with friends via SNS platforms required real-time tracking. The uLike method allowed us to automatically track the uploaded posts and shared data in real-time, thus we were able to obtain the information to measure viral effects for uLike in Step 1 of Phase I. For the nonuLike method, however, we were unable to track the uploaded posts because the method was not equipped for that function. Therefore, in this phase, we decided to collect the viral effect for non-uLike posts using the official Facebook page that could track the uploaded posts and shared data (e.g., likes, comments).

Fo obtain such data, we posted event contents on the official Facebook page for 24 hous, and we collected the viral data. For this experiment, the subjects were the fans who joined the 2014 Hwacheon Festival's official Facebook page during Phase I of Step 1 as shown in Figure 6. The total number of subjects was 56, consisting of 49 people using the uLike method and 7 people using the non-uLike method. Because these subjects had already posted content about the 2014 Hwacheon Festival 2014 on their SNSs through Phase I, event information was exposed and shared by their SNS friends.

Thus, in phase II, to have a fair comparison, we needed a different event instead of the event used for Phase I. Accordingly, we decided to use the 2014 Hwacheon Jjokbae Festival because the size of that marketing event was similar to the Hwacheon Sancheoneo Ice Festival, and the events were held in the same area and had the same host. 
The goal of this event was to promote the festival that ran from July $26^{\text {th }}$ to August $10^{\text {th }}$ in 2014 with online SNS posts. The Hwacheon Jjokbae Festival has been held every summer in Hwacheon-gun, South Korea, and the main program is creating a uniquely shaped boat and keeping it afloat on the Hwacheon River. Table 5 summarizes information about phase II of the experiment.

Table 5. The Experiment Information for Phase II

\begin{tabular}{ll}
\hline \hline Experiment Event & Pre-promotion event for the 2014 Hwacheon Jjokbae Festival \\
\hline Experiment Date & May 24, 2014, at 11 am \\
\hline Experiment Place & Online \\
\hline Experiment Subjects & $\begin{array}{l}56 \text { fans who joined the official Facebook page of the 2014 Hwacheon } \\
\text { Sancheoneo Ice Festival during Step 1 in phase I }\end{array}$ \\
\hline Experiment Method & The marketing event using the official Facebook page as a non-uLike method \\
\hline \hline
\end{tabular}

\subsection{Data Collection}

This section provides a brief description of the qualitative and quantitative data collected during the experiment. A total of 134 people participated in our experment: 83 participants used uLike, and 51 participants used a non-uLike method. We wanted the same number of participants for each method, but we yere not able to control this factor due to the following reason; the non-uLike method required people to install a mobile SNS application to proceed with the experiment, but some participants decided not to install the application on their mobile phone and withdrew from the experiment.

The quantitative data included uLike posting results wiral factors, and non-uLike posting results (i.e., Step 1 in phase I)! Also, it included viral factors from the official Facebook page (i.e., Phase II). The qualitative dataincluded participants' feedback about and ratings (on a 5-point Likert scake) for different characteristics related to the usability of the uLike method (i.e., Step 2 ir (Phase I).

\subsection{Data Analysis and Results}

This section presents the results of our experiment and the data analysis for each research question.

\subsubsection{Online-Exposure Effectiveness (RQ1)}

Our first research question (RQ1) considered whether using uLike can improve the online exposure effectiveness that indicates increased business exposure. To answer RQ1, we measured the number of posts, the number of posts per posting type (text, photo, and video clip), and the number of posts per participant. Figure 7(a) shows the total number of posts for the uLike and non-uLike methods; Figure 7(b) shows the number of posts per posting type and Figure 7(c) shows the number of posts per participant.

Some observations from Figure 7 are as follows:

- The total number of posts by the 83 participants who used uLike was 218; the number of posts for text, photos, and video clips was 75, 71, and 72, respectively; and the number of posts per participant was 2.672. On the other hand, the total number of posts by non-uLike participants was 72 ; the number of postings for text, photos, and video clips was 14, 51, and 7, respectively; and the number of posts per participant was 1.286.

- Because the number of uLike and non-uLike participants was quite different (uLike: 83 and non-uLike: 51), it was not fair to compare the total number of posts, so we evaluated the number of posts per participant. As shown in Figure 7(c), the number of posts for uLike users was twice as high as non-uLike users. These results indicated that utilizing $u$ Like increased business exposure because it encouraged customers to upload more posts than non-uLike participants. 


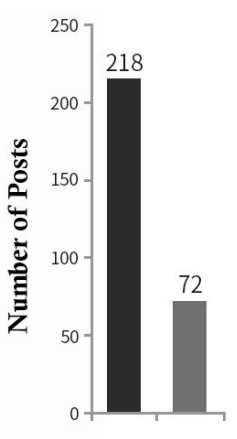

(a)

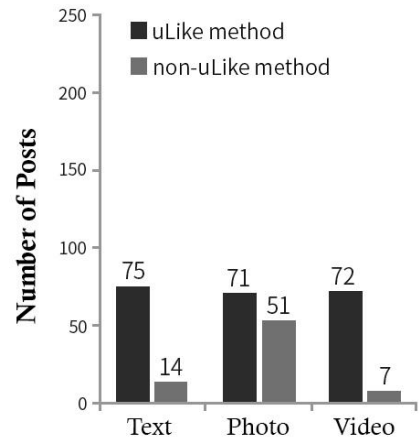

(b)

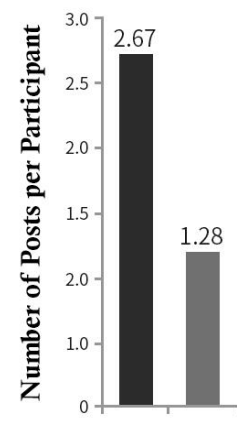

(c)

Figure 7. Comparison for the Number of Posts

Figure 8. Comparison for humber of Fans

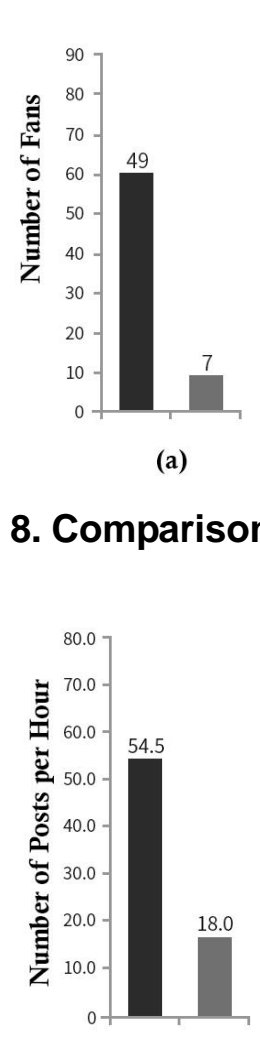

(a)

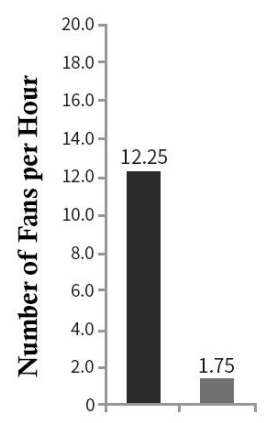

(b)

Figure 9. Comparison of the Efficiency 


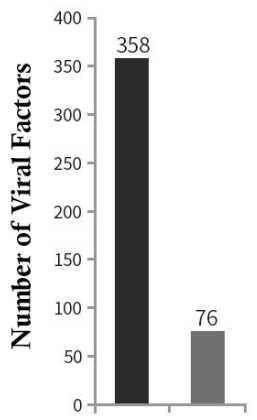

(a)

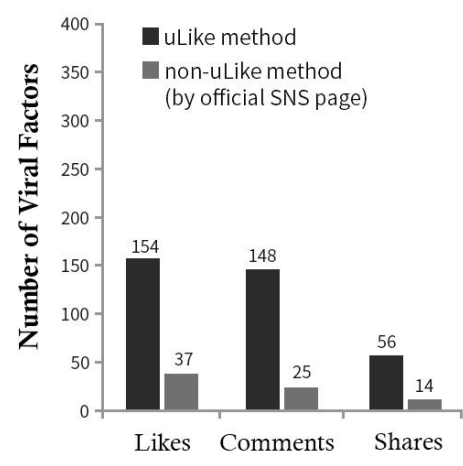

(b)

Figure 10. Comparison of the Viral Effect

- As shown in Figure 7(b), for the uLike method, the number of posts was similar across all posting types. One possible reason for this finding could be that it was easier for participants to upload preconfigured content On the other hand, with the non-uLike method, the number of posts varied by the posting type- Kexts (19.44\%), photos (70.83\%), and video clips (9.72\%). More than two-thirds of the posts were photos. A possible reason for this could be that, in the case of texts and video clips, they were somewhat cumbersome to create because the participants needed to typewrite themselves while uploading photos was easier because the people just needed to take pictures with their own mobile device

We also measured the number of fans who subscribed to the content of the official Facebook page. Figure 8 shows the total number of lans and the number of fans per participant for the uLike and non-uLike experiments.

Some observations from Figure 8 are as follows:

- The number of fans for the official Facebook page with the uLike method was 49 while the non-uLike method only had 7 fans as shown in Figure 8(a). Because the number of uLike and non-uLike participants was different (uLike: 83 and nonuLike: 51), to have a fair comparison, we assessed the number of fans per participant As shown in Figue 8(b), the number collected by uLike is 4 times higherthan that with the non-uLike (uLike: 0.59 and non-uLike: 0.14 ).

- This result indicated that uLike increased business exposure by producing more fans than the non-ulike method.

\subsubsection{Online Exposure Efficiency (RQ2)}

Our second research question (RQ2) considered whether utilizing uLike improved the online-exposure efficiency, indicating usability and accessibility. To answer RQ2, we measured tyo values: the number of posts per hour and the number of fans per hour.

Some observations from Figure 9 are as follows:

Figure 9(a) shows the number of posts per hour for the uLike and non-uLike methods. The number for uLike is almost three times higher than that for non-uLike (uLike: 54.5 and non-uLike: 18.0). In the case of uLike, the simple action of tagging specified readers can easily and quickly post preconfigured texts, photos, and video clips. These results indicate that utilizing $u$ Like increases the posting speed.

- Figure 9(b) shows the numbers of fans per hour for the uLike and non-uLike methods. The number for uLike is almost four times higher than that for non-uLike (uLike: 0.59 and non-uLike: 0.14 ). With the uLike method, participants do not need to connect to the official page to become a fan as long as they click "like" in the process of issuing an NFC tag. In contrast, for the non-uLike method, participants need to connect to the official page to click "like" in order to become a fan, which 
might discourage them from participating. These results indicate that utilizing $u L i k e$ increased the number of fans for an official SNS page more efficiently than the non-uLike method.

\subsubsection{Viral Effect (RQ3)}

Our third research question (RQ3) considered whether using uLike can improve the viral effect of online marketing; this effect was associated with the follow-up activities taken by the participants' SNS friends. To answer RQ3, we measured the number of viral factors, such as likes, comments, and shares. Figure 10(a) shows the total number of viral factors, and Figure 10(b) shows the number of viral factors per viral-factor type.

Some observations from Figure 10 are as follows:

- The total number of viral factors produced by SNS friends of the 83 uLike participants was 358 , and the numbers of viral factors for likes, comments, and shares was 154,148 , and 56, respectively. On the other hand, the total number of viral factors produced by SNS friends of the 56 fans of the official SNS page (nonuLike) was 76, and the number of viral factors for likes, comments, and shares was 37,25 , and 14 , respectively.

- When we compared the number of viral factors per participant the number for uLike was almost three times higher than that for the official SNS page (non-uLike) (uLike: 4.31 and non-uLike 1.36). These results indicated that using uLike produces higher viral effects than the official SNS page and that ollow-up marketing activities can be enhanced with uLike.

\subsubsection{Usefulness of uLike (RQ4)}

uLike users and non-uLike users provided feedback through a survey that asked about six attributes: innovativeness, usability, attractiveness, brand-image promotion, future uLike use, and overall satisfaction. Figure 11 shows the survey results.

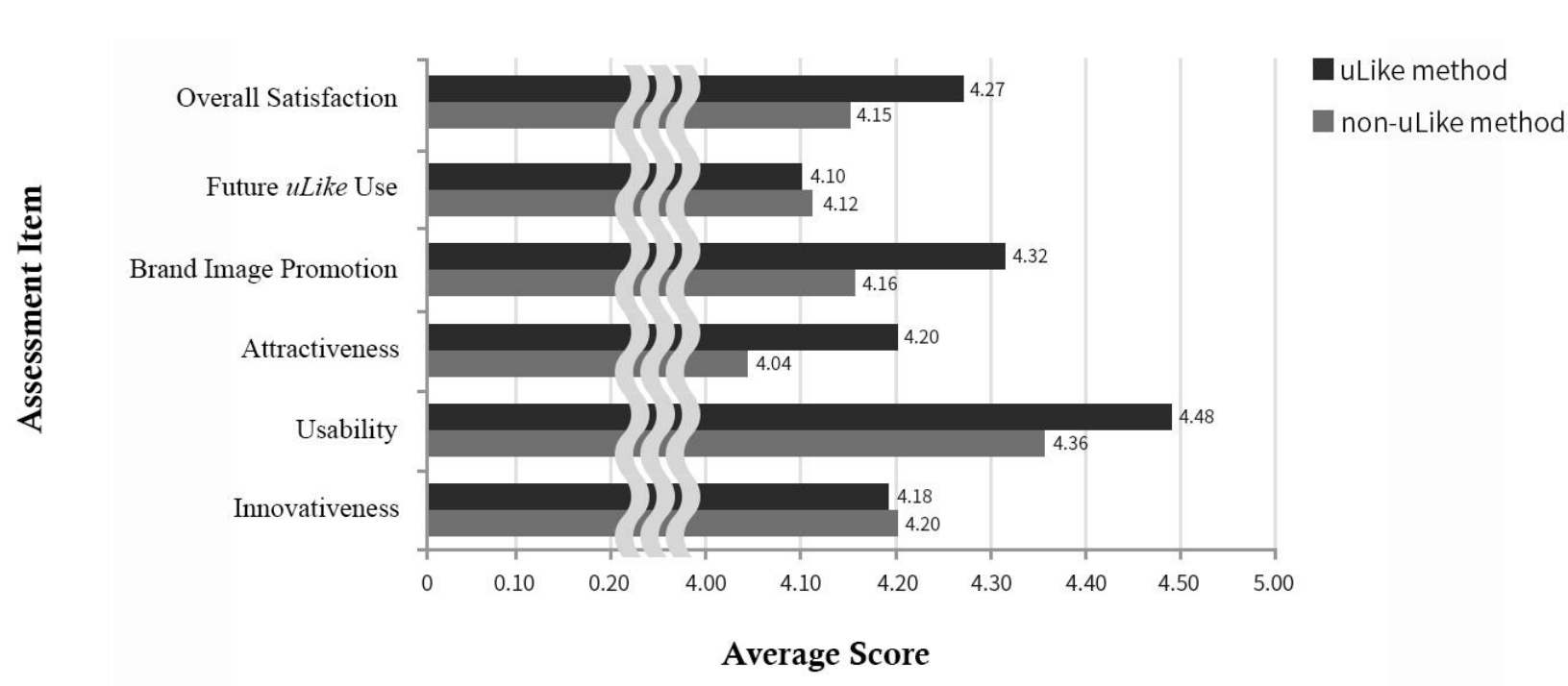

Figure 11. Assessment Results 
Participants were asked to indicate the degree of agreement or disagreement with each attribute on a 5-point Likert scale. Each scale item had 5 response categories, ranging from strongly agree to strongly disagree (1, Strongly disagree; 2, Disagree; 3, Indifferent; 4, Agree; or 5, Strongly agree).

Some observations from Figure 11 are as follows:

- The averages of the five-point scale for each evaluation item for people who experienced $u L i k e$ were $4.18,4.48,4.20,4.32,4.10$, and 4.27 , respectively. The average of these six values was 4.26. Meanwhile, the averages of the five-point scale for each evaluation item for people who did not experience uLike but received an explanation about it with a story board were 4.20, 4.36, 4.04, 4.16, 4.12, and 4.15, respectively. The average for these six values was 4.17.

- These results indicated that both the participants who have experienced and heard about uLike favored the uLike system.

- Usability had the highest score among the evaluation items for both groups." Therefore, the usability of uLike (The simple action of tagging automatically performs all the procedures of posting.) was its most appealing feature.

\subsection{Threats to Validity}

In this study, there were some threats to validity that were addressed. First, the number of participants for two methods was not balanced because the non-uLike method required installing mobile application of SNS, but we tried to-reduce this threat by allotting equal experiment time for both groups and by randomly assigning participants to each group. Second, if the contents for two methods are different, the result will be biased. In order to resolve this threat, we used the same content for the experimental and comparison groups. Third, the inference we made about the effectiveness of the $u$ Like method could have been affected by potential faults with the wike tool. To control this threat, we validated the uLike tool using several pilot studies

However, there were some threats that were not addressed. The official Facebook page used for our experiment was created by one of the authors, so it might not represent official industry pages Besides, we did not artificially control the number of SNS friends for the participants in order to keep the viral effect natural. The experimental results about the viral effects could have been affected by the participants' number of SNS friends. These threats-can be addressed through more studies, and we plan to resolve these threats in the future. Further, toreduce the external threats to validity, we plan to apply the uLike method with various offline, events that have diverse participants.

\section{Discussion of Results}

This section illustrates the results and their implications in light of the original research questions. The findings and the lessons from this study are then discussed.

Online Exposure Effectiveness (RQ1): Our results indicated that uLike can be more effective for increasing the exposure of online advertisements than the traditional SNS offlime marketing-event tool. The major findings were as follows: (1) the number of posings with uLike was much larger than with the non-uLike method. (2) uLike produced more fans for the official SNS page than the non-uLike method. The uLike method enabled the participants to post content and to become a fan of the official page more easily because it was linked to the SNS using NFC technology. Moreover, it was possible to collect the offline data produced during the events and to track the data online in real time. However, participants who used the non-uLike method needed extra time and effort to post contents because the non-uLike method was not linked SNS platforms. Further, there were limitations with collecting and tracking data from offline events for the nonuLike method. In Step 1 of Phase I, for example, we were not able to obtain data regarding the viral factors because the method was not equipped to track such information. 
Online Exposure Efficiency (RQ2): Our results indicated that uLike can be more efficient for increasing the exposure of online advertisements than the traditional SNS offline marketing-event tool. The major findings were as follows: (1) the number of posts per hour for uLike was 3 times higher than for non-ulike users. (2) The number of fans per hour with uLike was 4.2 times higher than with the non-uLike method. We speculated that uLike's ease of use affected these outcomes. uLike allowed the participants to post content to their SNS platforms by touching the RFID tag to a reader while the non-uLike method required content to be created and posted manually. Further, by using preconfigured content, $u$ Like reduced the time needed to create content.

Viral Effect (RQ3): Our results indicated that uLike can be more effective for continuous, word-of-mouth and voluntary public relations than the existing SNS online marketing-event tool. The number of viral factors per participant with uLike was 3.2 times higher than for participants with the non-uLike method. For our method, personalized content was posted through the participants' news feed, and the content was about their own experiences at offline events. We believe that these facts can increase attention and responses from SNS friends. Therefore, the SNS friends voluntarily shared and spead the content, and these activities increased the viral marketing effect. On the other hand, with official-page-centered events by companies, which are the typical type of existing social media marketing, companies post marketing-oriented content on their official pages. These posts are not personalized, so the users are discouraged from shanng or spreading the contents.

Usefulness of uLike (RQ4): Our results indicated that uLike can be more useful to encourage the customers to participate in the events. Most people responded that our method is innovative and easy to use compared to the existing social media marketing method. Further, the participants perceived the event with uLike positively, and they responded that they are willing to parficipate futuresevents that use uLike.

\section{Conclusions}

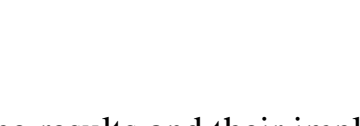

This section illustrates the results and their implications in light of the original research questions. The findings and the lessons from this study are then discussed. We presented a new offline based marketing method linked with social media using NFC technology, uLike (a social media marketing service that we developed and that implements our approach), and its infrastructure. We presented an empirical study that was performed using uLike to investigate our method's effectiveness and efficiency. The results of the study showed that the uLike method can be more effective and efficient for increasing online exposure and can be more useful than the existing social media marketing method.

We discussed the limitations of our study in Section 4.5. To address those limitations and to reduce threats to validity, we plan to conduct additional experiments including the use of other types of social media marketing methods. We also plan to improve the $u$-Like method by providing a mechanism that can generate personalized contents for posting. Through the results reported in this paper, and the future work, we hope to provide companues that rely on social media for their business a better understanding of the benefits the uLike method offers. Furthermore, we hope this work can motivate companies to adopt $u$ Like for their marketing events and thus they can increase business opportunities and improve business strategies. 


\section{Acknowledgements}

The authors would like to thank to members of the uLikeKorea Co., Inc. for their support. This work was funded by the MSIP (Ministry of Science, ICT, and Future Planning), Korea, under the ITRC (Information Technology Research Center) support program (NIPA-2014-H0301-14-1023) supervised by NIPA (National IT Industry Promotion Agency). This work was also supported, in part, by NSF CAREER Award CCF-1564238 to University of North Texas.

\section{References}

[1] K. Lerman, "Social Information Processing in News Aggregation", Internet Computing, IEEE, vol. 11, no. 6, (2007), pp. 16-28.

[2] D. Kiron, D. Palmer, A. N. Philips and N. Kruschwitz, "Social Business: What Are Companies Really Doing", MIT Sloan Management Review, vol. 53, no. 4, (2012), pp. 1-32.

[3] D. M. Boyd and N. B. Elison, "Social Network Sites: Definition, History, and Scholarship", Journal of Computer-Mediated Communication, vol. 13, no. 1, (2007), pp. 210-230.

[4] W. G. Mangold and D. J. Faulds, "Social Media: The New Hybrid Element of the Promotion Mix", Business horizons, vol. 52, no. 4, (2009), pp. 357-365.

[5] K. Hersant, "9 Best Practices of Social Media Marketing”, Software World, vol. 2, no. 6, (2011), pp. 21-22.

[6] M. A. Stelzner, "Social Media Marketing Industry Report. Hom Marketers Are Using Social Media to Grow Their Businesses", Social Media Examiner, (2015) pp.7

[7] P. Mourdoukoutas and G. J. Siomkos, "Seven Principles of WOM and Buzz Marketing", Springer Science \& Business Media, (2009).

[8] E. Keller and J. Berry, "Word-of-mouth: The Real Action Is Offline", Advertising Age, vol. 77, no. 49, (2006), pp. 20-28.

[9] H. Kim and B. Choi, "O2O-based Social Medi Marketing Method for Word-Of-Mouth Effect: Focused on the Analysis of Case Studies", Journal of the Korea Contents Association, vol. 15, no. 7, (2015), pp. 403-413.

[10] F. Michahelles, F. Thiesse, A. Schmidt and J. R. Williams, "Pervasive RFID and Near Field Communication Technology", IEEE Peryasive Computing, vol. 6, no. 3, (2007), pp. 94-96.

[11] NFC Forum, NDEF: NFC Data Exchange Format, Reyision 1.0, www.nfc-Forum.org/specs/, (2006).

[12] http://www.ulike.kr

[13] A. Rampell, "Why Online2Offline Commerce Is A eTrillion Dollar Opportunity”, TechCrunch, (2010).

[14] R. Hanna, A. Rohm and V. L. Crittenden. "We're All Connected: The Power of the social media ecosystem”, Business horizons, vol. 54, no. 3, (2011), pp. 265-273.

[15] http://www.youtube.com/watch?v=P-zA90yI64

[16] W. Xingang and L. Zhang, "Analysis of O2O Model's Development Problems and Trend", iBusiness, vol. 7, no. 1, (2015), pp. 51-57.

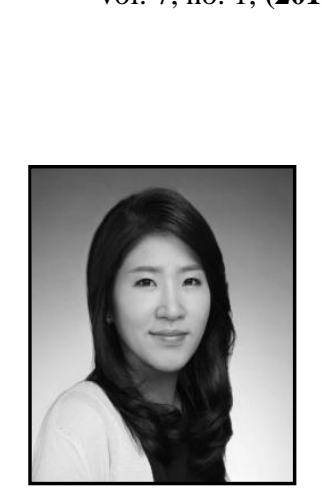

\section{Authors}

Heejin Kim, is working toward the Ph.D. degree in the Department of Computer Science and Engineering at Ewha Womans University and is in charge of the representative director of the uLikeKorea Co., Inc. in South Korea. She received her B.S. and M.S. degrees in computer science from Ewha Womans University. Her research interests include software engineering with particular emphasis on cloud computing and IoT Technology.

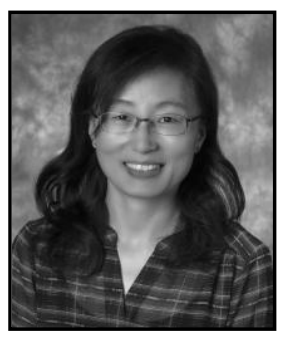

Hyunsook Do, is an associate professor in the Department of Computer Science and Engineering at University of North Texas in United States. She received the Ph.D. in Computer Science from University of Nebraska-Lincoln, the M.S. in Computer Science from Tokyo Institute of Technology in Japan and a B.S. in Computer Science from Sungshin Women's University in South Korea. Her 


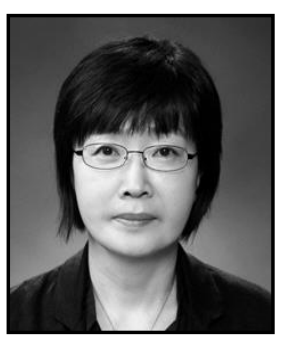

research interests lie in software engineering, particularly software testing and empirical methodologies.

Byoungju Choi is a full professor in the Department of Computer Science and Engineering at Ewha Womans University in South Korea. She received a B.S. degree in mathematics from Ewha Womans University, M.S. and Ph.D. degrees in computer sciences from Purdue University. Her research interests include software engineering with particular emphasis on cloud computing, software testing, embedded software testing, software and data quality.

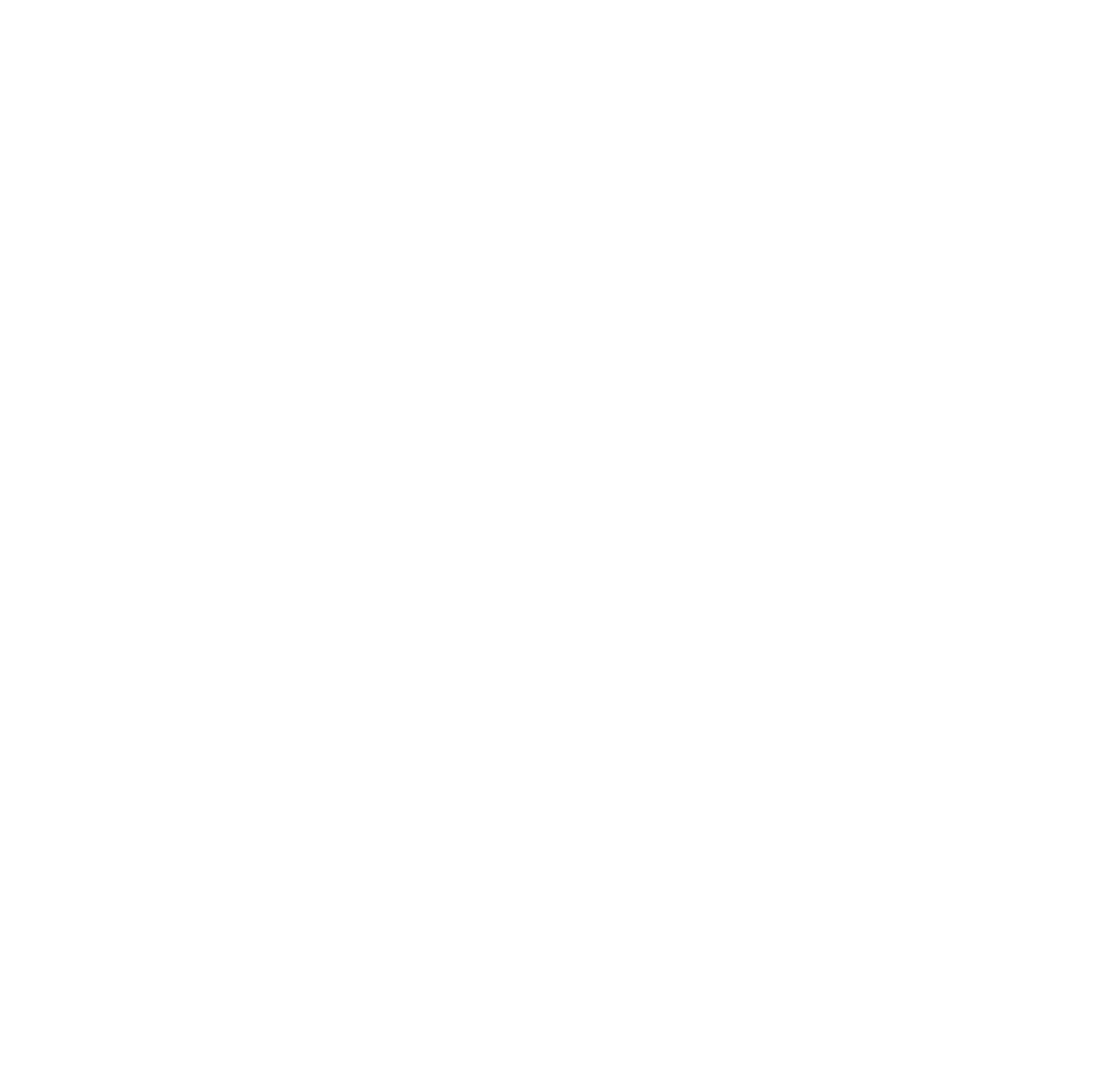


International Journal of Multimedia and Ubiquitous Engineering

Vol.11, No.11 (2016)

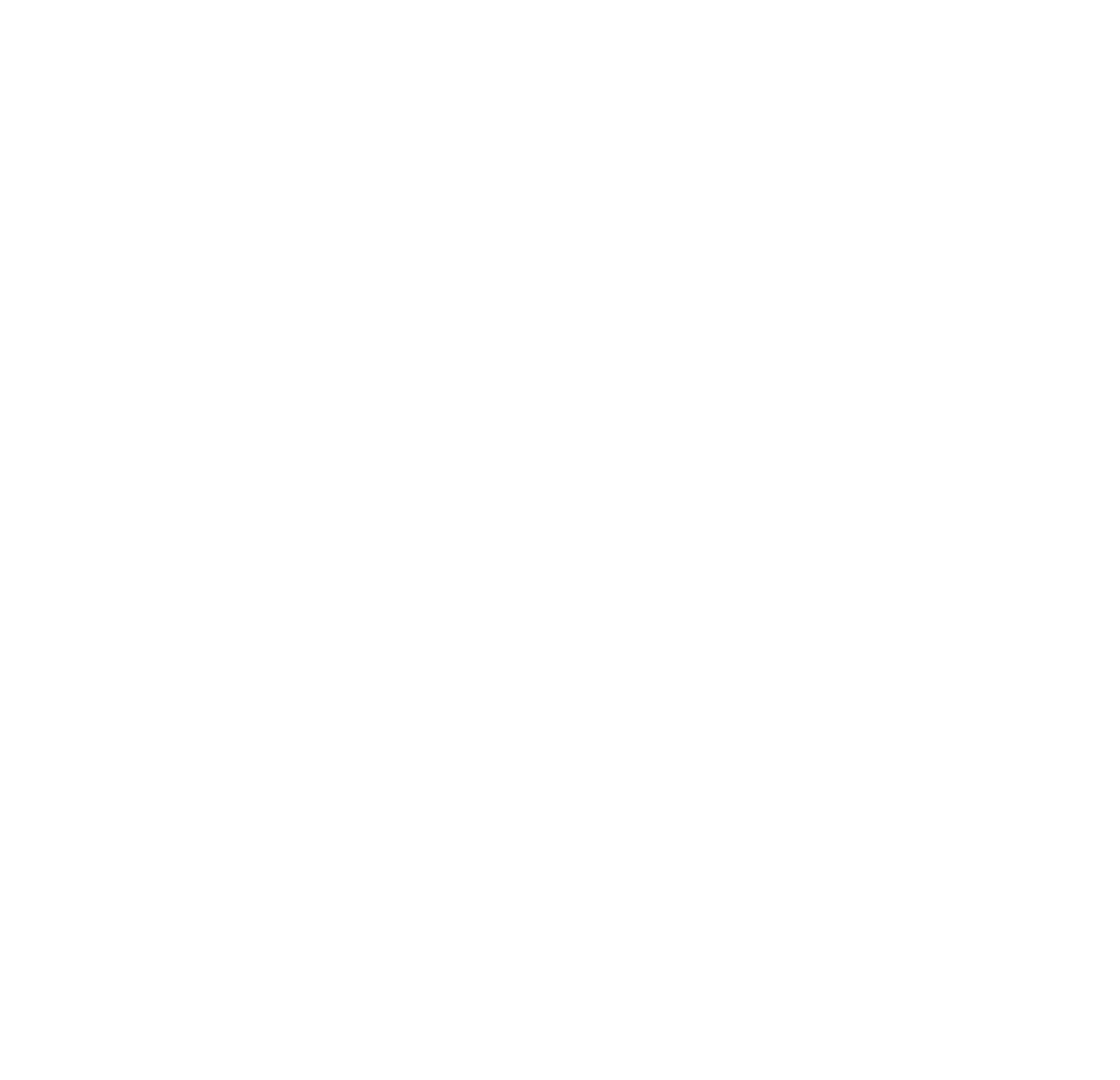

